\title{
Oscillations With Mechanical Insufflation-Exsufflation in ALS: Time to Pause and Return to Basics?
}

Mechanical insufflation-exsufflation (MI-E) is a cough augmentation therapy used primarily in patients with respiratory muscle weakness. In amyotrophic lateral sclerosis (ALS), MI-E is a first-line treatment irrespective of bulbar function. In a broad spectrum of neuromuscular conditions, MI-E enhances cough peak flow and is associated with improved respiratory outcomes such as reduced rates of infection and hospitalization. ${ }^{1,2}$ Over the last decade, MI-E device development has provided the ability to deliver personalized care by adjusting pressure, flow, and the ratio of insufflations to exsufflations, along with the addition of oscillations. The evidence base for the benefit of adding oscillations to MI-E therapy remains limited.

Oscillation therapies are thought to improve mucociliary clearance by enhancing expiratory flow and cilia beat frequency, and through reducing sputum viscosity. ${ }^{3}$ However, evidence for use of oscillation in the neuromuscular population is sparse. ${ }^{4}$ Sancho and colleagues ${ }^{5,6}$ are leaders in investigating the additive effect of oscillations with MI-E. An early prospective study of 47 stable subjects with ALS measured cough peak flow under 4 combinations of MI-E and oscillation: without oscillation, with oscillation during insufflation, with oscillation during exsufflation, and with oscillation during insufflation/exsufflation cycles. The study failed to demonstrate any impact of oscillations on cough peak flow. ${ }^{5}$ Recently, these authors reported on the impact of oscillations on the need for invasive secretion management procedures in 29 stable patients with ALS. This prospective randomized trial, conducted over a 12month period, concluded that the addition of oscillations had no effect on the need for bronchoscopy or tracheostomy in this cohort. ${ }^{6}$

In this issue of the Journal, Sancho and colleagues ${ }^{7}$ report the results of a randomized crossover trial investigating the addition of oscillations to MI-E in 19 subjects with ALS who received home tracheostomy ventilation. Subjects received their usual MI-E therapy or MI-E with oscillations, for 6 months each, in a randomized order. Primary outcomes included rate of respiratory infection, hospital admissions, and need for bronchoscopy over the 12-month period. There

The authors have disclosed no conflicts of interest.

Correspondence: Louise Rose PhD. E-mail: louise.rose@kcl.ac.uk. DOI: $10.4187 /$ respcare.08954 were no differences in the outcomes between the 2 treatment modalities, and the authors concluded that the addition of oscillations to MI-E for subjects with ALS and home tracheostomy ventilation did not reduce the risk of respiratory infection or bronchoscopy.

See the Original Study on Page 378

The authors should be commended for completing trials in such a complex and difficult to research patient group. However, we believe the authors' conclusion that oscillations added to MI-E provide no benefit is incautious due to several methodological flaws that we outline here. First, a sample size calculation is not reported, but one can assume that a sample of 19 subjects is unlikely to provide the required statistical power to make such conclusions. The authors note in their study limitations that the total number of patients with ALS managed with home tracheostomy ventilation is small. Thus, while pragmatic study designs are necessary, limitations in interpreting their results must be acknowledged. Second, subjects were described as "clinically stable." However, the authors reported that tenacious secretions remained problematic despite a routine airway clearance regime including MI-E. Readers must consider, therefore, whether MI-E settings and other sputum management strategies were optimized before oscillations were introduced. Third, insufflation and exsufflation pressures used were $\pm 40 \mathrm{~cm} \mathrm{H}_{2} \mathrm{O}$. These are the recommended starting pressures for patients receiving MI-E via a face mask. ${ }^{4}$ Bench data indicate that an artificial airway reduces peak flow during MI-E due to increased airway resistance, which suggests that higher delivered pressures are needed to overcome this resistance and generate a peak flow that is clinically effective. ${ }^{8}$ Therefore, the insufflation and exsufflation pressures of $\pm 40 \mathrm{~cm} \mathrm{H}_{2} \mathrm{O}$ used in this study of subjects managed with home tracheostomy ventilation may have been insufficient.

Chatwin and Toussaint ${ }^{9}$ recently summarized the evidence base for the use of oscillations with MI-E and concluded that, although there are no existing data to support the clinical use of oscillations in MI-E, many outstanding questions need to 


\section{EDITORIALS}

be addressed. Before more studies are conducted on the use of oscillations with MI-E, the importance of optimizing secretion management in patients with ALS must be emphasized. Several parameters can be manipulated on MI-E devices, but our knowledge of the utility of these parameters is limited primarily to bench studies. ${ }^{10,11}$ Before considering oscillations, the physiological and clinical impact of each MI-E parameter needs to be better understood. The ultimate aim is to produce evidence-based guidance on each MI-E parameter to allow clinicians to deliver personalized care depending upon the clinical condition of the patient.

Effective secretion clearance may also require mucolytics, nebulizers, and appropriate mouth care and feeding/hydration regimes, in addition to cough augmentation techniques. The contributory and potentially confounding effect of these cointerventions should not be ignored in future clinical trials.

Much of the clinical data that exist on MI-E have methodological flaws, primarily due to the small sample sizes available to individual institutions and the large effect size needed to demonstrate clinical efficacy. To provide definitive answers on the benefits of MI-E with or without oscillations, future research needs multi-center and multinational collaboration. Until we are delivering optimal MI-E in the absence of oscillations as part of a comprehensive secretion management plan, we anticipate that studies investigating the addition of oscillations to MI-E will struggle to draw firm conclusions as to benefit or lack thereof.

Ema Swingwood

Adult Therapy Services

University Hospitals Bristol and Weston NHS Foundation

Trust

Bristol, United Kingdom

Neeraj M Shah

Lane Fox Clinical Respiratory Physiology Centre Guy's and St Thomas' NHS Foundation Trust London, United Kingdom
Louise Rose

Florence Nightingale Faculty of Nursing, Midwifery and Palliative Care

King's College London

London, United Kingdom

\section{REFERENCES}

1. Chatwin M, Ross E, Hart N, Nickol AH, Polkey MI, Simonds AK. Cough augmentation with mechanical insufflation/exsufflation in patients with neuromuscular weakness. Eur Respir J 2003;21(3):502-508.

2. Morrow B, Zampoli M, van Aswegen H, Argent A. Mechanical insufflation-exsufflation for people with neuromuscular disorders. Cochrane Database Syst Rev 2013;12:CD010044.

3. Moores C, Hudson NE, Davies A. The effect of high-frequency ventilation on non-Newtonian properties of bronchial mucus. Respir Med 1992;86(2):125-130.

4. Chatwin M, Toussaint M, Gonçalves MR, Sheers N, Mellies U, Gonzales-Bermejo J, et al. Airway clearance techniques in neuromuscular disorders: a state of the art review. Respir Med 2018;136:98-110.

5. Sancho J, Bures E, de La Asunción S, Servera E. Effect of High-frequency oscillations on cough peak flows generated by mechanical inexsufflation in medically stable subjects with amyotrophic lateral sclerosis. Respir Care 2016;61(8):1051-1058.

6. Sancho J, Burés E, Ferrer S, Bondía E, Servera E. Usefulness of oscillations added to mechanical in-exsufflation in amyotrophic lateral sclerosis. Respir Care 2020;65(5):596-602.

7. Sancho J, Burés E, Ferrer S, Lahosa C, Signes-Costa J, Servera E. Mechanical insufflation-exsufflation with oscillations in amyotrophic lateral sclerosis with home ventilation via tracheostomy. Respir Care 2021;66(3):378-383.

8. Guérin C, Bourdin G, Leray V, Delannoy B, Bayle F, Germain M, et al. Performance of the coughassist insufflation-exsufflation device in the presence of an endotracheal tube or tracheostomy tube: a bench study. Respir Care 2011;56(8):1108-1114.

9. Chatwin M, Toussaint M. Will the addition of oscillations in mechanical insufflation-exsufflation ever be beneficial? Respir Care 2020;65 (5):725-728

10. Gómez-Merino E, Sancho J, Marín J, Servera E, Blasco ML, Belda FJ, et al. Mechanical insufflation-exsufflation: pressure, volume, and flow relationships and the adequacy of the manufacturer's guidelines. Am J Phys Med Rehabil 2002;81(8):579-583.

11. Volpe MS, Naves JM, Ribeiro GG, Ruas G, Amato MBP. Airway clearance with an optimized mechanical insufflation-exsufflation maneuver. Respir Care 2018;63(10):1214-1222. 